\title{
Simulação do impacto do fungo Nomuraea rileyi em populações da lagarta da soja, Anticarsia gemmatalis ${ }^{(1)}$
}

\author{
Edison Ryoiti Sujii(2), Myrian Silvana Tigano ${ }^{(2)}$ e Daniel Sosa-Gomes ${ }^{(3)}$
}

\begin{abstract}
Resumo - O fungo entomopatogênico Nomuraea rileyi (Farlow) Samson produz epizootias em populações de lagarta da soja, Anticarsia gemmatalis (Huebner), controlando naturalmente a praga em determinadas condições. No entanto, a epizootia nem sempre ocorre a tempo de evitar que as populações desta praga causem dano econômico à cultura. Foi criado um modelo matemático para simular a ocorrência de $N$. rileyi em populações de A. gemmatalis nas lavouras de soja da Flórida, EUA. O objetivo deste trabalho foi desenvolver um sistema que integrasse esse modelo a outros modelos (fenologia da soja e da praga, interações inseto-planta e dinâmica populacional da praga), a fim de gerar simulações mais precisas no manejo da praga. Estes modelos foram construídos a partir de estudos ecológicos dos organismos envolvidos, conduzidos nas áreas de soja no Brasil. O sistema integrado foi desenvolvido com base em equações de diferenças que são processadas pelo programa STELLA, versão 5.0 Research. A avaliação do modelo em Planaltina, DF, e Londrina, PR, demonstraram que o sistema é capaz de simular a ocorrência de epizootias ou explosões populacionais da lagarta da soja.
\end{abstract}

Termos para indexação: Insecta, fungo entomopatogênico, epizootia, combate às pragas.

\section{Simulation of the impact of the fungus Nomuraea rileyi on populations of velvetbean caterpillar, Anticarsia gemmatalis}

\begin{abstract}
The entomopathogen fungus Nomuraea rileyi (Farlow) Samson may produce epizooties in populations of velvetbean caterpillar, Anticarsia gemmatalis (Huebner) under specific conditions. However, sometimes the epizooty does not occur in time to avoid that populations of the pest reach economic threshold level in the soybean crop. A mathematical model was created to simulate occurrence of $N$. rileyi on populations of A. gemmatalis in soybean crops of Florida, USA. The objective of this work was to develop a system to integrate this original model with several other models (phenology of pest and crop, insect plant interactions, and population dynamics of the pest), in order to generate more accurate simulations on the pest management. These models were developed from ecological studies involving organisms in soybean fields of Brazil. The integrated system was proposed based on equations of differences that are processed as differential equations through the software STELLA, version 5.0 Research. The evaluation of the system using data from soybean fields from Planaltina, DF, and Londrina, PR, showed that the model was able to simulate occurrence of epizooties or pest outbreaks in field conditions.
\end{abstract}

Index terms: Insecta, entomogenous fungi, epizooty, pest management.

\section{Introdução}

O fungo entomopatogênico Nomuraea rileyi (Farlow) Samson é conhecido como agente de

\footnotetext{
(1) Aceito para publicação em 15 de julho de 2002.

(2) Embrapa-Centro Nacional de Pesquisa de Recursos Genéticos e Biotecnologia, Caixa Postal 02372, CEP 70849-970 Brasília, DF. E-mail: sujii@cenargen.embrapa.br, myrian@cenargen.embrapa.br

(3) Embrapa-Centro Nacional de Pesquisa de Soja, Caixa Postal 231, CEP 86001-970 Londrina, PR.

E-mail: sosa@cnpso.embrapa.br
}

controle biológico de lepidópteros-praga de diversas culturas. Existem cerca de 30 espécies de lepidópteros já registradas como suscetíveis a este fungo entomopatogênico, com epizootias em lagartas que atacam várias culturas, pastagens e plantas daninhas (Ignoffo et al., 1976). As lagartas da família Noctuidae estão entre as mais suscetíveis a este patógeno, e sob certas condições ambientais este fungo é capaz de reduzir drasticamente populações destes insetos nos EUA, Brasil, Argentina e Austrália (Corrêa \& Smith, 1975; Carner, 1980; Ignoffo, 1981; Lecuona, 1990). 
A consistência de ocorrência e os altos índices de controle natural obtidos com $N$. rileyi sugerem a consideração deste como um componente importante de programas de manejo integrado de pragas. No Brasil, entre os hospedeiros suscetíveis, destaca-se Anticarsia gemmatalis (Huebner), praga da cultura de soja (Kogan et al., 1977). A presença de $N$. rileyi impede que a população de A. gemmatalis atinja o nível de dano econômico, evitando assim a aplicação de inseticidas contra essa praga. Nas culturas de soja do centro-sul do Brasil, $N$. rileyi pode causar epizootias em populações de A. gemmatalis (Faria et al., 1993). Porém, a ocorrência de períodos de baixa precipitação nos meses de janeiro e fevereiro pode impedir que este fenômeno ocorra a tempo de evitar que as populações desta praga atinjam o nível de dano econômico (Tigano-Milani et al., 1995).

Os modelos matemáticos que simulam a dinâmica populacional de insetos-praga, e o seu impacto na produção, têm sido largamente utilizados, principalmente em culturas anuais como a soja (Mishoe et al., 1984; Nagarajan et al., 1994; Gazzoni et al., 1998). Um modelo desenvolvido por Kish \& Allen (1978), na região da Flórida, EUA, simula o impacto da ocorrência natural de $N$. rileyi em populações de A. gemmatalis.

O objetivo deste trabalho foi desenvolver, a partir de um modelo matemático, um sistema integrado de modelos para as relações no sistema planta/inseto/fungo a fim de gerar simulações mais precisas no manejo da praga.

\section{Material e Métodos}

Foram monitoradas populações de lagartas de A. gemmatalis nos anos agrícolas 1996/97 e 1998/99 em áreas de $2.500 \mathrm{~m}^{2}$, cultivadas com soja na região de Planaltina, DF, plantadas com as cultivares Doko e Celeste, respectivamente. No ano agrícola 1997/98, foram realizadas amostragens em uma área de $2.660 \mathrm{~m}^{2}$ cultivada com soja (cultivar BR 37), em Londrina, PR.

Em 1996, o levantamento foi realizado em intervalos regulares, que variaram de quatro a sete dias. A amostragem consistiu da inspeção de todas as plantas em $1 \mathrm{~m}$ de linha de soja para verificar a presença de lagartas, com dez amostras sorteadas ao acaso a cada data.
A fim de ajustar a entrada de dados e resultados do modelo ao método de amostragem com pano de batida (Kogan et al., 1977), os levantamentos das populações de lagartas, em 1998 e 1999, foram realizados por esse método, a intervalos de dois a cinco dias, durante o ciclo da cultura.

As lagartas sadias e mortas amostradas foram classificadas por tamanho: pequenas ( $1^{\circ}$ e $2^{\circ}$ ínstares) e grandes ( $3^{\circ}, 4^{\circ}, 5^{\circ}$ e $6^{\circ}$ ínstares). Nesta classificação, as lagartas grandes incluíram aquelas consideradas médias e grandes no modelo original. As lagartas mortas por infecção incluíram as mumificadas, as recobertas por micélio ou por conídios de N. rileyi (Kish et al., 1976).

Os dados climáticos foram obtidos durante os levantamentos, em estações meteorológicas localizadas na Embrapa-Centro Nacional de Pesquisa de Soja, no Paraná, e na Embrapa-Centro de Pesquisa Agropecuária dos Cerrados, no Distrito Federal, distantes 2 e $5 \mathrm{~km}$ do campo experimental, respectivamente.

O modelo matemático para simular a ocorrência de $N$. rileyi em populações de A. gemmatalis, na região da Flórida, EUA, desenvolvido por Kish \& Allen (1978), considera a densidade de lagartas presentes na soja e relaciona a produção e dispersão dos conídios com a ocorrência de fatores ambientais, segundo as relações expressas nas equações abaixo:

$\mathrm{PC}=\left(\operatorname{LP} \times 5,6 \times 10^{8}\right)+\left(\operatorname{LM} \times 1,5 \times 10^{9}\right)+\left(\operatorname{LG} \times 3,18 \times 10^{9}\right)$, onde: PC é a produção potencial de conídios; LP é o número de lagartas pequenas $(<16 \times 1,5 \mathrm{~mm})$; LM é o número de lagartas médias ( $<25$ x 2,5 mm); LG é o número de lagartas grandes $(<34 \times 3,5 \mathrm{~mm})$;

$\mathrm{IA}=(\mathrm{PC})(\mathrm{FA})(\mathrm{UR} \times \mathrm{PR} \times \mathrm{VE} \times \mathrm{UV} \times \mathrm{FF})(\mathrm{AF})^{-1}$, onde: IA é o inóculo no ambiente dado em conídios $/ \mathrm{mm}^{2}$ de área foliar; FA é o fator de adesão dos conídios ao cadáver; UR é o efeito da umidade relativa; PR é efeito da precipitação; VE é o efeito do vento; UV é o efeito da radiação ultra-violeta; FF é o fator de perda por falta de cobertura foliar; $\mathrm{AF}$ é a área foliar por hectare $\mathrm{em}^{\mathrm{mm}^{2}}$.

No processo de desenvolvimento do modelo, a equação original, que ajusta as taxas de infecção da lagarta em função da densidade de inóculo do fungo no ambiente (Ignoffo et al., 1976), foi substituída pelas curvas obtidas de Boucias et al. (1984) e Moscardi et al. (1992), sucessivamente, para melhorar o ajuste do modelo às observações de campo.

As densidades de lagartas sadias e infectadas obtidas nos levantamentos de campo foram inseridas no modelo para calcular a proporção de lagartas mortas. O modelo considera um período fixo de seis dias para o desenvolvimento da infecção (Kish \& Allen, 1978). As taxas observadas no campo e simuladas pelo modelo foram comparadas graficamente e através da análise de correlação de Pearson (Wilkinson, 1990). 
Considerando as variações na correlação entre as simulações e as coletas de campo em diferentes anos e visando aumentar a precisão do modelo para as condições brasileiras, informações sobre fenologia, interação inseto/planta hospedeira e ciclo de vida do inseto, constantes no modelo proposto por Gazzoni et al. (1998), foram usadas na construção de um sistema integrado, para simular em computador a dinâmica populacional de $A$. gemmatalis e as taxas de infecção causadas pelo fungo.

O sistema integrado foi composto de quatro modelos que funcionam de modo interdependente. Cada modelo recebe dados relativos a fatores ambientais e características do agroecossistema durante o período da simulação e retroalimentam os modelos relacionados (Figura 1). O sistema foi desenvolvido com base em equações de diferenças que são processadas em computador pelo programa STELLA, versão 5.0 Research, como equações diferenciais, gerando funções contínuas.

Os modelos descrevem os seguintes processos:

1) Soja e interações inseto-planta - O modelo é baseado no estágio fenológico da planta de soja de acordo com o desenvolvimento da área foliar, característico de cada cultivar utilizada. As simulações desenvolvidas no presente trabalho foram feitas com base na cultivar Paraná (Gazzoni et al., 1998), porém as curvas e a caracterização fenológica devem ser feitas para cada cultivar. A chegada de adultos migrantes à área de soja, a mortalidade de lagartas por

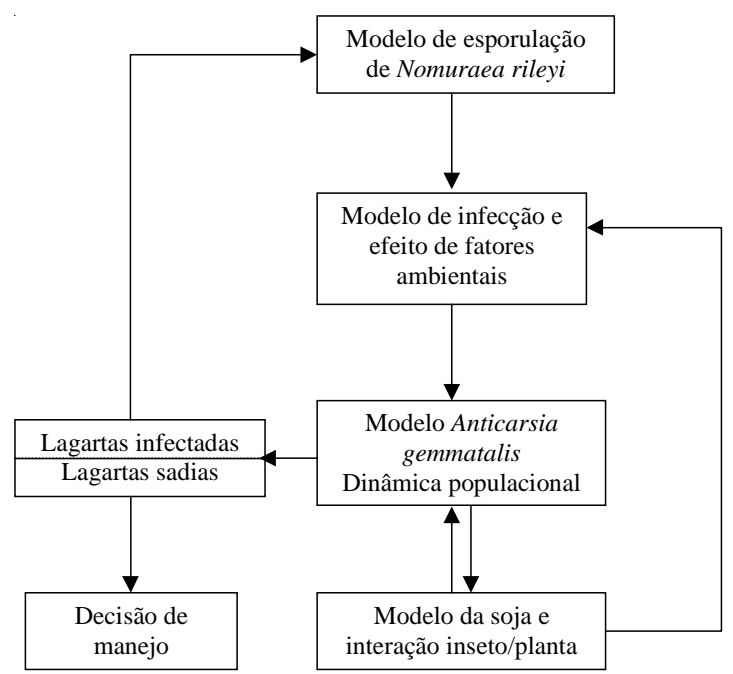

Figura 1. Fluxo de dados e interação entre os modelos componentes do sistema integrado para simulação da dinâmica populacional de Anticarsia gemmatalis, e da epizootia provocada pelo fungo Nomuraea rileyi. falta de alimentos e a taxa de produção de ovos pelas fêmeas são influenciadas pelo índice de área foliar e o estágio fenológico da cultura (Kogan \& Herzog, 1980). Assim, informações sobre a cultivar plantada, data de plantio e espaçamento, são consideradas pelos modelos nas simulações. Este modelo interage com o modelo do ciclo de vida do inseto, recebendo informações sobre a população de lagartas em cada estádio, enviando informações sobre o nível de desfolha produzido por estas lagartas e a disponibilidade de recurso alimentar (Figura 2).

2) Ciclo de vida e bionomia de A. gemmatalis A quantidade instantânea de indivíduos em cada estágio de desenvolvimento de A. gemmatalis é calculada neste modelo segundo a equação geral de diferenças:

$\mathrm{N}_{\mathrm{st}}=\mathrm{R}_{(\mathrm{s}-1)}+\mathrm{S}_{\mathrm{s}}-\mathrm{D}_{\mathrm{s}} \pm \mathrm{M}_{\mathrm{s}}$, onde: $\mathrm{N}_{\mathrm{st}}$ é o número de indivíduos no estágio s e tempo t; $\mathrm{R}_{(\mathrm{s}-1)}$ é o número de indivíduos recrutados do estágio s-1 entre $\mathrm{t}-1 \mathrm{e} \mathrm{t} ; \mathrm{S}_{\mathrm{s}}$ é o número de indivíduos remanescentes do estágio $\mathrm{s}$ no tempo t-1; $\mathrm{D}_{\mathrm{s}}$ é o número de indivíduos mortos no estágio s no tempo $\mathrm{t}-1 ; \mathrm{M}_{\mathrm{s}}$ é o número de indivíduos migrantes no estágio s entre $\mathrm{t}-1$ e t.

O ciclo de vida do inseto, com a estrutura populacional por estágio de desenvolvimento e os fatores de mortalidade, é apresentado de modo diagramático na Figura 3. As densidades absolutas de cada população são calculadas a partir do levantamento no campo das primeiras lagartas, dos ovos produzidos pelos primeiros adultos migrantes observados no campo, do efeito da temperatura média, do índice de área foliar no desenvolvimento e da sobrevivência das lagartas e capacidade reprodutiva dos adultos. Os dados sobre a população de adultos migrantes e índice de área foliar são informados pelo modelo de soja e da interação inseto-planta. A temperatura média diária é informada ao sistema mediante uma tabela de dados meteorológicos em um arquivo anexo (planilha eletrônica), com ligação dinâmica entre os programas STELLA (simulador) e QUATTRO PRO (planilha).

Entrada de dados da soja

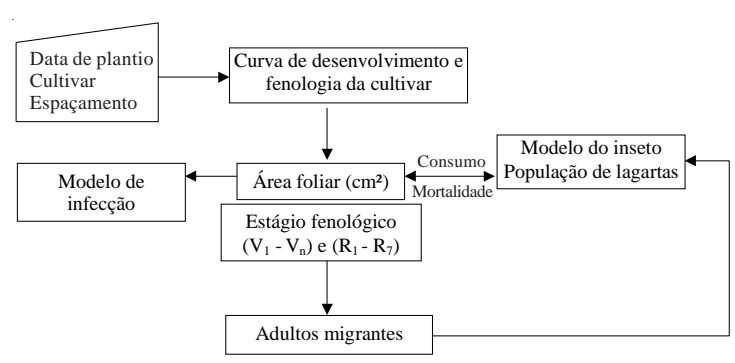

Figura 2. Fluxograma do modelo de desenvolvimento da planta de soja e sua integração com outros modelos. Estágios fenológicos da soja: $\mathrm{V}_{\mathrm{n}}$ : vegetativo; $\mathrm{R}_{\mathrm{n}}$ : reprodutivo. 
Os dados sobre o ciclo de vida do inseto, o consumo foliar diário pelas lagartas, a fecundidade das mariposas e as taxas de mortalidade decorrentes da predação, parasitismo, interação inseto/planta e temperatura, são aqueles utilizados por Gazzoni et al. (1998). As taxas de fecundidade e eclosão de lagartas e adultos, em razão da temperatura média, foram ajustadas graficamente por interpolação linear a partir das tabelas propostas por Gazzoni et al. (1998). As simulações resultantes desse modelo geraram as curvas de flutuação populacional dos diferentes estágios de desenvolvimento do inseto.

3) Esporulação de $N$. rileyi sobre cadáveres de A. gemmatalis - A concentração do inóculo primário de $N$. rileyi no campo é informada ao modelo através do monitoramento da população de lagartas no campo. Amostragens periódicas, com o auxílio do pano de batida, foram feitas para determinar a data e densidade inicial das lagartas infectadas pelo fungo, no campo, e o início do processo de desenvolvimento da doença, na área.

A quantidade potencial de conídios que podem ser produzidos diariamente é determinada neste modelo pela distribuição de tamanhos das lagartas mortas por N. rileyi (Figura 4):

$P C=\left[\left(\operatorname{LP} \times 10,3 \times \mathrm{e}^{8}\right)+\left(\operatorname{LG} \times 3,18 \times \mathrm{e}^{9}\right)\right] \times(\mathrm{ES})^{-1}$, onde: PC é a produção diária de conídios; LP é o número de lagartas pequenas infectadas $(\mathrm{L} 1+\mathrm{L} 2)$; $\mathrm{LG}$ é o número de lagartas grandes infectadas (L3 + L4 + L5 + L6 $\times 2^{-1}$ ); ES é o espaçamento da soja.

O processo de esporulação do fungo é também afetado por fatores abióticos. O tempo entre a exposição da lagarta ao fungo e a formação de micélio na superfície do cadáver varia de quatro a seis dias, de acordo com o tamanho da lagarta. A partir desse ponto, a formação de conídios

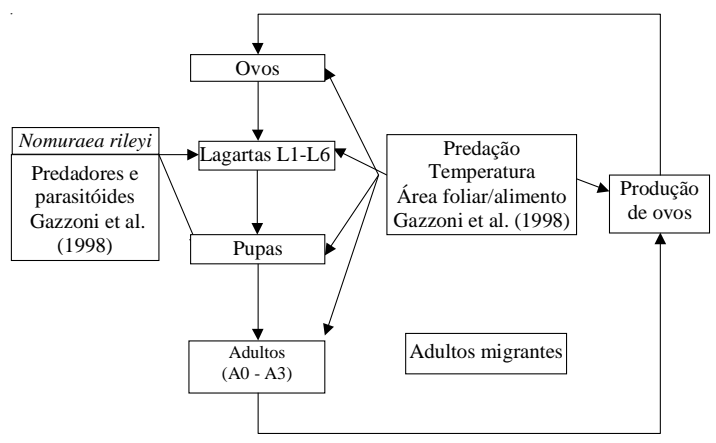

Figura 3. Fluxograma do modelo simulador da dinâmica populacional de Anticarsia gemmatalis. A0: adultos préreprodutivos; A1 a A3: adultos maduros; L1 a L6: estádios durante a fase de lagarta. se inicia entre um e quatro dias e se prolonga por até seis dias, quando ocorre o máximo da esporulação. A ocorrência de umidade relativa do ar acima de $80 \%$ por mais de 16 horas por dia, em dias consecutivos, favorece o início do processo de esporulação e a produção de mais conídios.

4) Infecção e epizootia - Fatores abióticos como precipitação, velocidade do vento, umidade relativa do ar e radiação ultra-violeta afetam a dispersão e a viabilidade dos conídios produzidos sobre os cadáveres, de acordo com a seguinte equação (Kish \& Allen, 1978):

$\mathrm{IA}=(\mathrm{PC})(\mathrm{FA})(\mathrm{UR} \times \mathrm{PR} \times \mathrm{VE} \times \mathrm{UV} \times \mathrm{FF})(\mathrm{AF})^{-1}$, onde: IA é o inóculo no ambiente dado em conídios $/ \mathrm{mm}^{2}$ de área foliar; PC é a produção diária de conídios; FA é o fator de adesão $=0,9$ (10\% dos conídios não desprendem do cadáver); UR é o efeito da umidade relativa $[(\mathrm{H}+$ 2PA) x $24^{-1}$ ]; H é o número de horas de umidade relativa acima de 80\%; PA é a precipitação no dia anterior; PR é o efeito da precipitação [(precipitação em $\mathrm{mm}$ ) $\times 6,3^{-1} \mathrm{x}$ $0,63]$; VE é o efeito do vento [(1 - $\left.\left.\left(\mathrm{F} \times 24^{-1}\right) \times 63,13^{-1}\right)\right]$; F é a velocidade do vento em $\mathrm{m} / \mathrm{min}$; UV é o efeito da radiação ultra-violeta $=0,5$ (50\% de inviabilidade); FF é o fator de perda por falta de cobertura foliar $\left(1-1 \mathrm{x} \mathrm{G}^{-1}\right)$; $\mathrm{G}$ é o índice de área foliar; $\mathrm{AF}$ é a área foliar por hectare em $\mathrm{mm}^{2}$.

A equação para simular a quantidade de conídios que é efetivamente depositada nas folhas e que serve como fonte de inóculo para calcular a proporção de lagartas mortas segundo a curva de infecção proposta por Moscardi et al. (1992) é a seguinte:

$\mathrm{LI}=2,60832+1,65613(\mathrm{IA})$,

onde: LI é o número de lagartas infectadas em \%; IA é o inóculo no ambiente dado em conídios $/ \mathrm{mm}^{2}$ área foliar.

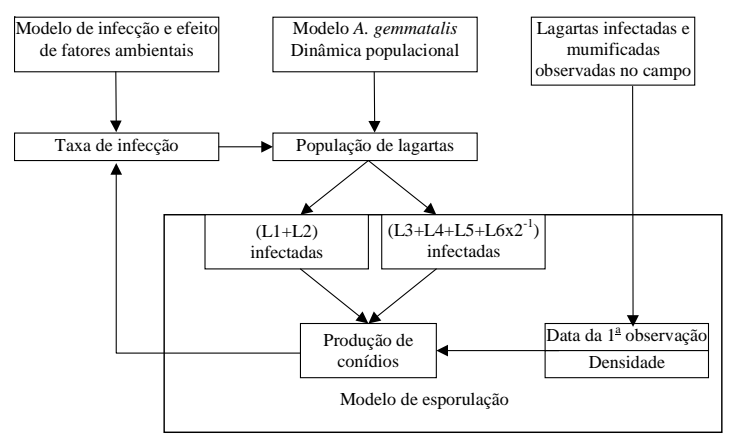

Figura 4. Modelo de esporulação de Nomuraea rileyi, e as interações com outros modelos do sistema. L1 a L6: estádios durante a fase de lagarta. 
As curvas de proporção de insetos infectados e mortos em virtude da ação de $N$. rileyi, e as curvas de flutuação populacional geradas pelo modelo de A. gemmatalis, são os principais resultados do sistema integrado de simulação (Figuras 1 e 5).

$\mathrm{Na}$ avaliação do sistema integrado, foram realizadas simulações utilizando os dados climáticos e da dinâmica populacional de A. gemmatalis dos anos agrícolas de 1996/97, 1997/98 e 1998/99. Os resultados das simulações foram comparados com levantamentos populacionais realizados nos respectivos anos, através do teste de Kolmogorov-Smirnov (Wilkinson, 1990).

\section{Resultados e Discussão}

Os monitoramentos das áreas de soja nas regiões de Planaltina, DF, e Londrina, PR, em diferentes anos, demonstraram que as populações de A. gemmatalis apresentam densidades populacionais mais elevadas em anos com baixa precipitação pluviométrica e com distribuição irregular durante o verão. O fungo $N$. rileyi ocorreu em todas as áreas, produzindo taxas de infecção características de curvas epizoóticas mesmo quando a densidade das lagartas esteve abaixo do nível de dano econômico de 20 ou 40 lagartas por pano de batida, conforme é preconizado pela Embrapa (1997). Esses dados confirmam e ampliam as observações de que $N$. rileyi geralmente ocorre em surtos epizoóticos anuais sobre populações da lagarta A. gemmatalis, atuando como agente de controle natural das populações do inseto em diferentes regiões produtoras de soja no país (Corrêa \& Smith, 1975; Faria et al., 1993).

As primeiras lagartas infectadas por $N$. rileyi no ano de 1996/97, em Planaltina, DF, foram observadas em nove de janeiro, quando a densidade populacional média era de 7,8 lagartas/amostra

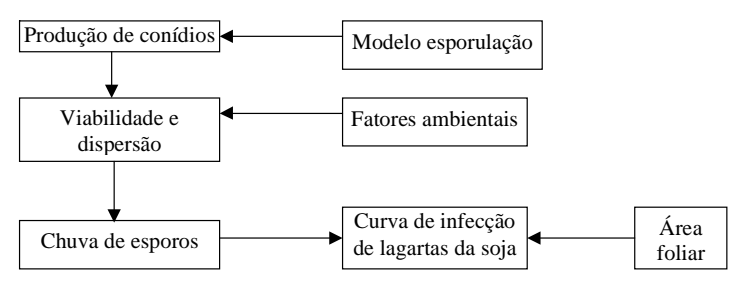

Figura 5. Modelo de infecção da lagarta, e efeito de fatores ambientais na viabilidade e dispersão de conídios de Nomuraea rileyi na cultura da soja.
(1 m de linha de soja plantada). A progressão da taxa de infecção foi mais lenta que o crescimento da população de lagartas, que chegou a atingir o nível de dano econômico de 40 lagartas pequenas/pano de batida antes de 19 de janeiro. Somente após 23 de janeiro, as taxas de infecção por $N$. rileyi estiveram acima de $80 \%$ (Figura 6).

No ano agrícola de 1997/98, na região de Londrina, PR, a população de lagartas não alcançou uma densidade populacional que justificasse seu controle (Figura 6). O período de dez dias sem precipitação, na segunda quinzena de janeiro, parece não ter afetado significativamente a ocorrência da epizootia.
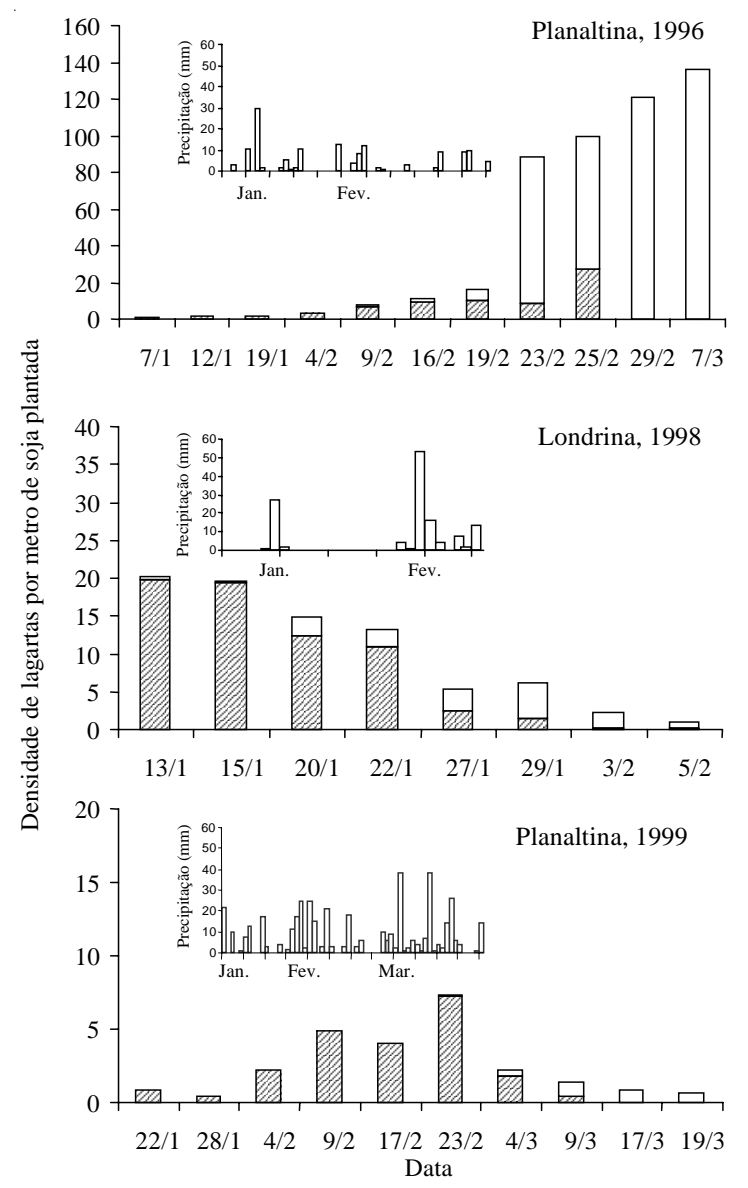

Figura 6. Flutuação populacional de lagartas de Anticarsia gemmatalis (অ) e taxa de infecção por Nomuraea rileyi ( $\square$ ) em área de soja na região de Planaltina, DF, em 1996, Londrina, PR, em 1998 e Planaltina, DF, em 1999.

Pesq. agropec. bras., Brasília, v. 37, n. 11, p. 1551-1558, nov. 2002 
Durante o ano agrícola de 1999, em Planaltina, DF, as condições de pluviosidade alta e bem distribuída durante o período de dezembro a fevereiro, aparentemente, impediram o aumento populacional do inseto. A partir de março, a densidade populacional de A. gemmatalis aumentou ligeiramente, porém não atingiu o nível de dano econômico, e permitiu o aumento do número de lagartas infectadas por $N$. rileyi em uma curva epizoótica (Figura 6).

A simulação resultante do modelo de Kish \& Allen (1978), no ano de 1996, apresentou uma relativa precisão entre os níveis de infecção observados e os previstos (coeficiente de correlação de Pearson $\mathrm{r}=0,87, \mathrm{t}=4,18, \mathrm{p}<0,05, \mathrm{n}=6$ ), à exceção do período crítico para o manejo da praga, 9 a 19 de fevereiro (Figura 6), quando o modelo superestimou as taxas de infecção (Figura 7). A substituição da equação para ajustar a curva de infecção pela equação proposta por Moscardi et al. (1992), utilizando isolados
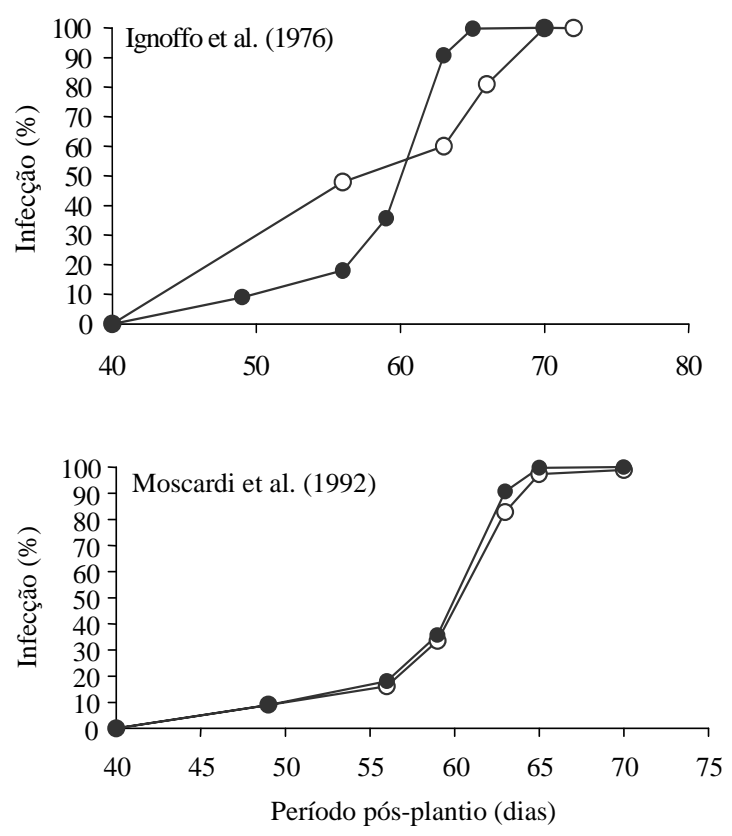

Figura 7. Taxas de infecção do fungo Nomuraea rileyi em lagartas de Anticarsia gemmatalis, na região do DF, em 1996, observadas no campo (•) e simuladas pelo modelo de Kish \& Allen (O) utilizando a curva de infecção proposta por Ignoffo et al. (1976) e Moscardi et al. (1992). brasileiros de $N$. rileyi, melhorou o ajuste às observações de campo $(r=0,998, t=33,75, p<0,001, n=6)$ e foi incluída no modelo (Figura 7).

A avaliação do modelo de Kish \& Allen (1978), simulando as taxas de mortalidade causada pelo fungo em populações de A. gemmatalis, em diferentes locais e anos, Londrina 1997/98 e Planaltina 1998/99, demonstrou a ocorrência de flutuações inconsistentes entre as taxas de infecção observadas no campo e as simuladas pelo modelo (respectivamente $\mathrm{r}=0,80, \mathrm{t}=4,09, \mathrm{p}<0,05, \mathrm{n}=8$ e $\mathrm{r}=0,87$, $\mathrm{t}=2,85, \mathrm{p}>0,05, \mathrm{n}=4$ ) (Figura 8). A inconsistência dos resultados obtidos foi atribuída, por hipótese, à ausência de informações sobre a fenologia da planta, história de vida do inseto, impacto de variáveis ambientais na dinâmica populacional e interações com a planta hospedeira no processo de simulação do modelo. Um sistema que considera as informações acima de forma integrada foi construído para testar essa hipótese.
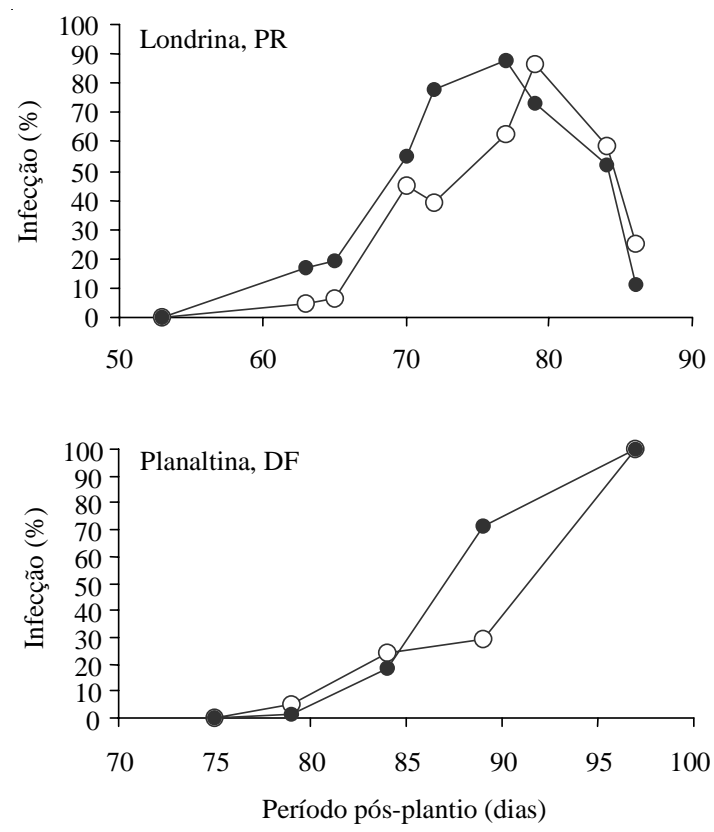

Figura 8. Taxas de infecção do fungo Nomuraea rileyi em lagartas de Anticarsia gemmatalis simuladas pelo modelo usando a curva de infecção de Moscardi et al. (1992) (O) e observadas no campo (•) em Londrina, PR, em 1998 e Planaltina, DF, em 1999. 
O monitoramento das populações de lagartas sadias e infectadas, ao longo de todo o ciclo da cultura, foi importante para determinar o provável fluxo de adultos migrantes após a germinação das sementes, a época de ocorrência e a densidade inicial das lagartas infectadas pelo fungo, e a época de densidade máxima de lagartas sadias e infectadas. Estes dados alimentaram os modelos e possibilitaram a avaliação das simulações produzidas pelo sistema integrado.

Não foram observadas diferenças significativas pelo teste de Kolmogorov-Smirnov, entre as curvas populacionais de lagartas sadias e mortas simuladas pelo sistema e observadas no campo em Planaltina, DF, em 1996 (diferença máxima padronizada $(M D)=0,3 ; p>0,05$ e $M D=0,26 ; p>0,05$, respectivamente), Londrina, $P R$, em 1998 ( $M D=0,333 ; p>0,05$ e $\mathrm{MD}=0,333 ; \mathrm{p}>0,05$, respectivamente) e Planaltina, $\mathrm{DF}$, em $1999(\mathrm{MD}=0,46 ; \mathrm{p}>0,05)$. As simulações resultantes do sistema apresentaram em geral um nível de precisão com diferenças inferiores a 33\% (Figura 9), quando comparadas aos levantamentos populacionais realizados em diferentes anos no Distrito Federal e Norte do Paraná. No entanto, quando ocorreram condições climáticas atípicas, como em 1999, com pluviosidade alta e bem distribuída e densidades populacionais extremamente baixas da lagarta, a diferença alcançou 46\% (Figura 9). A baixa densidade populacional de A. gemmatalis observada nessa ocasião tornou desnecessária qualquer medida de controle, não sendo necessário o uso do simulador.

A partir do fornecimento de dados da soja (data de plantio, variedade, espaçamento) ao sistema, além do monitoramento das condições climáticas e da densidade de lagartas sadias e infectadas, é possível traçar simulações, com antecedência de uma semana, sobre as populações esperadas de lagartas em áreas de soja. No entanto, somente após ajustes através de validações regionais e melhoria na precisão, mediante estudos complementares sobre a interação inseto/patógeno e o efeito de fatores ambientais, será possível fazer recomendações técnicas gerais ou regionalizadas aos produtores de soja sobre o manejo de A. gemmatalis, utilizando o controle natural por N. rileyi.

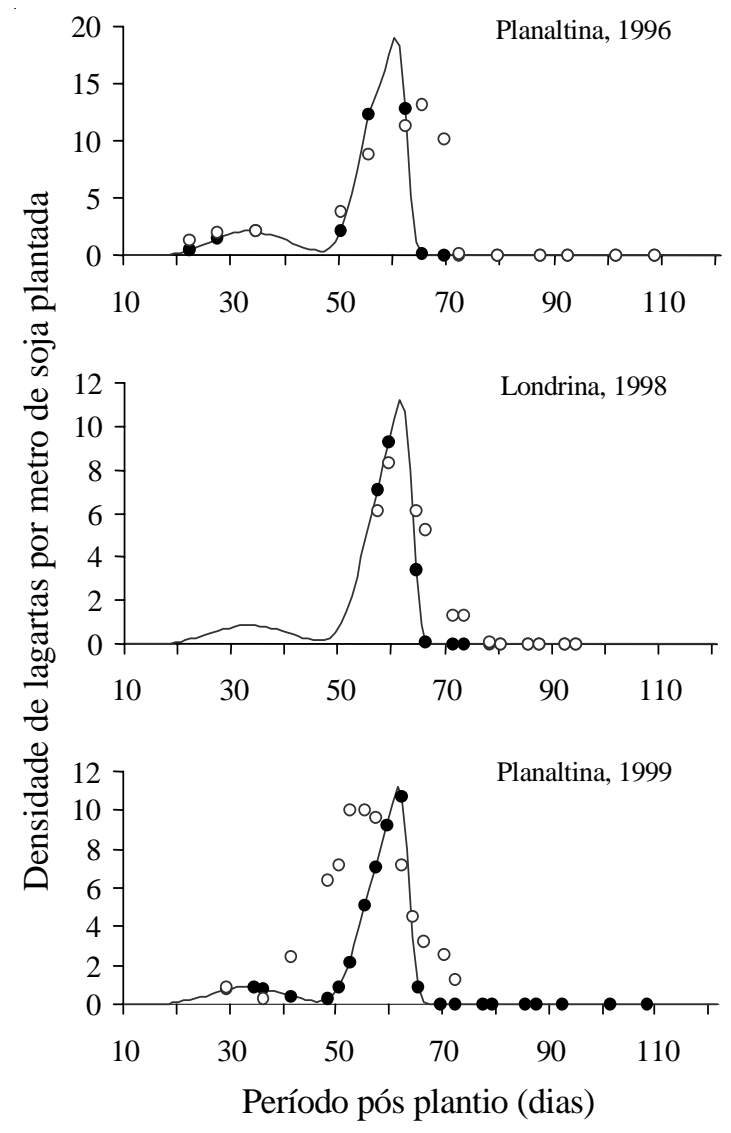

Figura 9. Comparação entre as densidades populacionais de Anticarsia gemmatalis simuladas pelo modelo $(\mathrm{O})$ e observadas no campo $(\bullet)$, em áreas de soja. As curvas de lagartas sadias (simulação) versus lagartas sadias (observadas no campo) não diferiram significativamente pelo teste de Kolmogorov-Smirnov.

\section{Conclusões}

1. Fatores ambientais, como fenologia da planta, história de vida e dinâmica populacional do inseto, além de interações inseto-planta, influenciaram o processo epizootiológico de $N$. rileyi em A. gemmatalis, e a inclusão desses processos na modelagem melhorou a precisão das simulações.

2. O sistema integrado é mais preciso que o modelo proposto por Kish \& Allen e é capaz de fazer simulações de densidade e época de ocorrência da praga, com desvios entre dados observados e resultantes da simulação inferiores a 30\%, quando há possibilidades de explosões populacionais da lagarta. 


\section{Agradecimentos}

A Irene Martins, Vanessa Andaló, Ana Teixeira e Rosângela Tiago, pela ajuda na coleta dos dados; ao Dr. Drion Boucias, pelas críticas e sugestões; à FAPDF e ao CNPq/Pronex, pelo apoio financeiro.

\section{Referências}

BOUCIAS, D. G.; BRADFORD, D. L.; BARFIELD, C. S. Susceptibility of the velvetbean caterpillar and soybean looper (Lepidoptera: Noctuidae) to Nomuraea rileyi: effects of pathotype, dosage, temperature and host age. Environmental Entomology, Lanham, v. 77, p. 247-253, 1984.

CARNER, G. R. Sampling pathogens of soybean insect pests. In: KOGAN, M.; HERZOG, D. C. (Ed.). Sampling methods in soybean entomology. New York: Springer, 1980. p. 559-574.

CORRÊA, B. S.; SMITH, J. G. Nomuraea rileyi attacking the velvetbean caterpillar, Anticarsia gemmatalis Huebner, in Paraná. Florida Entomologist, Homestead, v. 58, p. 280, 1975.

EMBRAPA. Centro Nacional de Pesquisa de Soja (Londrina, PR). Recomendações técnicas para a cultura da soja na região central do Brasil 1997/98. Londrina, 1997. 171 p. (Documentos, 106).

FARIA, M. R.; TIGANO-MILANI, M. S.; LECUONA, R. E. Incidência natural de Nomuraea rileyi Farlow em população de Anticarsia gemmatalis Huebner no Distrito Federal. Anais da Sociedade Entomológica do Brasil, Porto Alegre, v. 22, p. 385-388, 1993.

GAZZONI, D. L.; PEDROSO JUNIOR, M.; GARAGORRY, F.; MOSCARDI, F. Mathematical simulation model of the velvetbean caterpillar. Pesquisa Agropecuária Brasileira, Brasília, v. 33, n. 4, p. 385-396, abr. 1998.

IGNOFFO, C. M. The fungus Nomuraea rileyi as a microbial insecticide. In: BURGERS, H. D. (Ed.). Microbial control of pests and plant diseases: 19701980. London: Academic, 1981. p. 513-538.

IGNOFFO, C. M.; PUTTLER, B.; HOSTETTER, D. L.; DICKERSON, W. A. Susceptibility of the cabbage looper, Trichoplusia ni, and the velvetbean caterpillar, Anticarsia gemmatalis, to several isolates of the entomopathogenic fungus Nomuraea rileyi. Journal of Invertebrate Pathology, San Diego, v. 28, p. 259-262, 1976.

KISH, L. P.; ALLEN, G. E. The biology and ecology of Nomuraea rileyi and a program for predicting its incidence on Anticarsia gemmatalis in soybean. Gainesville: University of Florida, 1978. p. 1-58. (Agriculture Experimental Station Technical Bulletin, 795).

KISH, L. P.; GREENE, G. L.; ALLEN, G. E. A method of determining the number of potential conidia-forming cadavers of Anticarsia gemmatalis infected with Nomuraea rileyi in a soybean field. Florida Entomologist, Homestead, v. 59, p. 103-106, 1976.

KOGAN, M.; HERZOG, D. C. Sampling methods in soybean entomology. New York: Springer, 1980. 587 p.

KOGAN, M.; TURNIPSEED, S. G.; SHEPARD, M.; OLIVEIRA, E. B. de; BORGO, A. Pilot insect pest management program for soybean in southern Brazil. Journal of Economic Entomology, Lanham, v. 70, p. 659663, 1977.

LECUONA, R. E. El control microbiano como regulador poblacional de insectos plagas. Castelar: Instituto Nacional de Tecnologia Agropecuária, 1990. 24 p. (Serie Agricultura Sostenible, 4).

MISHOE, J. W.; JONES, J. W.; SWANEY, D. P.; WILKERSON, G. G. Using crop and pest models for management applications. Agricultural Systems, Oxford, v. 15 , p. $153-170,1984$.

MOSCARDI, F.; KASTELIC, J. G.; SOSA-GÓMEZ, D. R. Suscetibilidade de três espécies de lepidópteros associados à soja a três isolados do fungo Nomuraea rileyi (Farlow) Samson. Anais da Sociedade Entomológica do Brasil, Porto Alegre, v. 21, p. 93-100, 1992.

NAGARAJAN, K.; O’NEIL, R. J.; EDWARDS, C. R.; LOWENBERG-DEBOER, J. Indiana soybean system model (ISSM): I Crop model adaptation. Agricultural Systems, Oxford, v. 43, p. 357-379, 1994.

TIGANO-MILANI, M. S.; FARIA, M. R.; LECUONA, R. E.; SARTORI, M. R.; ARIMA, E. Y.; DIAZ, B. M. Análise de patogenicidade e germinação do fungo Nomuraea rileyi (Farlow) Samson isolado no Distrito Federal. Anais da Sociedade Entomológica do Brasil, Londrina, v. 24, p. 53-60, 1995.

WILKINSON, L. SYSTAT: the system for statistics. Evanson: SYSTAT, 1990. 676 p. 